\title{
Pyropia yezoensis glycoprotein promotes the M1 to M2 macrophage phenotypic switch via the STAT3 and STAT6 transcription factors
}

\author{
JEONG-WOOK CHOI ${ }^{1}$, MI-JIN KWON ${ }^{2}$, IN-HYE KIM ${ }^{2}$, YOUNG-MIN KIM ${ }^{1}$, \\ MIN-KYEONG LEE ${ }^{1}$ and TAEK-JEONG NAM ${ }^{1,2}$ \\ ${ }^{1}$ Department of Food and Life Science, Pukyong National University, Busan 608-737; \\ ${ }^{2}$ Institute of Fisheries Science, Pukyong National University, Busan 619-911, Republic of Korea
}

Received February 15, 2016; Accepted June 21, 2016

DOI: $10.3892 / \mathrm{ijmm} .2016 .2656$

\begin{abstract}
Macrophage polarization has been well documented. Macrophages can aquire two phenotypes, the pro-inflammatory M1 phenotype, and the anti-inflammatory and wound healing M2 phenotype. The M1 macrophage phenotype has been linked to metabolic disease and is also associated with cancerrelated inflammation. Of note, macrophage polarization can be influenced by the extracellular environment. In the current study, we examined the effects of Pyropia yezoensis glycoprotein (PYGP) on M1 to M2 macrophage polarization in lipopolysaccharide (LPS)-stimulated macrophages. RAW 264.7 macrophages stimulated with LPS exhibited an upregulated expression of pro-inflammatory mediators, namely of the M1 markers, nitric oxide (NO), reactive oxygen species (ROS), interleukin (IL)-6, IL-1 $\beta$, tumor necrosis factor- $\alpha$ (TNF- $\alpha$ ), interferon- $\gamma$ (IFN- $\gamma)$ and nitric oxide synthase-2 (NOS-2). Treatment with PYGP inhibited the production of M1 markers and increased arginase 1 (ARG1), chitinase-like 3 (Chil3; also known as Ym1), resistin like beta (RETNLB; also known as FIZZ1), IL-10, CD163, CD206, peroxisome proliferatoractivated receptor $\gamma$ (PPAR $\gamma$ ) and Krüppel-like factor 4 (KLF4) M2 marker gene expression. The signal transducer and activator of transcription (STAT)3 and STAT6 transcription factors were phosphorylated following treatment with PYGP. However, the silencing of STAT3 and STAT6 using siRNA in the macrophages decreased ARG1, Ym1 and FIZZ1 M2 marker gene expression in spite of treatment of PYGP. These findings suggest that PYGP exerts anti-inflammatory effects by regulating the M1 to M2 phenotypic switch through STAT3 and STAT6. Thus, PYGP may have potential for use as a natural remedy for inflammatory diseases.
\end{abstract}

Correspondence to: Professor Taek-Jeong Nam, Department of Food and Life Science, Pukyong National University, 599-1 Daeyeon 3-Dong, Nam-gu, Busan 608-737, Republic of Korea

E-mail: namtj@pknu.ac.kr

Key words: macrophage polarization, Pyropia yezoensis, signal transducer and activator of transcription 3, signal transducer and activator of transcription 6

\section{Introduction}

Macrophages are well-known not only as major regulators of innate and adaptive immunity, but also important mediators of systemic metabolism, hematopoiesis, vasculogenesis, apoptosis, malignancy and reproduction (1-4). There are two differentiation patterns, M1 and M2. M1 macrophages (classically activated macrophages) act as regulators of the host defense system. They protect from infection due to bacteria, protozoa and viruses (5). M2 macrophages (alternatively activated macrophages) have been reported to have anti-inflammatory activity and are important in wound healing (6). This plasticity can change according to the macrophage environment.

M1 activation is induced by interferon- $\gamma($ IFN- $\gamma)$ and lipopolysaccharide (LPS). The M1 phenotype upregulates proinflammatory cytokines and chemokines [e.g., tumor necrosis factor- $\alpha$ (TNF- $\alpha$ ), interleukin (IL)-12, IL-6, IL-1 $\beta$ and CCL2] and promotes the production of reactive oxygen and nitrogen species (ROS and RNS) $(8,9)$. LPS is well known as a stimulant for macrophages, and it is recognized for the activation of the Toll-like receptor 4 (TLR4)-related signaling pathway. TLR4 leads to the activation of the MyD88 and MaL/Tirap -dependent pathways, leading to the rapid switch to the M1 phenotype (10). The secretion of cytokines and chemokines in macrophages with the M1 phenotype is related to various transcription factors, such as nuclear factor- $\mathrm{B}(\mathrm{NF}-\kappa \mathrm{B})$, activator protein 1 (AP-1), interferon-regulatory factors (IRF)s and signal transducer and activator of transcription (STAT)1 (11). M1 macrophages have been reported to play an important role in chronic inflammatory diseases. Consequently, the abnormal or long-term activation of macrophages must be controlled to prevent damage to the host.

M2 activation is related to Th2-produced IL-4 and IL-13 (12). M2 macrophages are associated with the upregulation of galactose receptor, mannose receptor-1, chitinase-like 3 (Chil3; also known as Ym1), resistin like beta (RETNLB; also known as FIZZ1) and arginase 1 (ARG1) (13). Different metabolic processes are induced between M1 and M2. In particular, L-arginine metabolizes to produce nitric oxide (NO) in M1 macrophages, but in M2 macrophages L-arginine metabolizes to produce polyamines (1).

Pyropia yezoensis (P. yezoensis; Rhodophyta, Bangiaceae) is widely used as a food in Korea, China and Japan. P. yezoensis 
had been used as a medicine for the treatment of emesis, diarrhea and hemorrhoids in oriental medicine (14). P. yezoensis protein has been reported to have angiotensin I converting enzyme inhibitory activities (15), and to exert anti-inflammatory (16) and liver protective effects against acetaminophen (17).

In this study, we examined the effects of $P$. yezoensis glycoprotein (PYGP) on M1 to M2 macrophage polarization in lipopolysaccharide (LPS)-stimulated macrophages. In particulary, we focused on the similarities of the biological functions of anti-inflammation and wound healing between $P$. yezoensis and M2 polarization.

\section{Materials and methods}

Cell culture. RAW 264.7 cells were obtained from the American Type Culture Collection (ATCC,Manassas, VA, USA). The cells were grown in Dulbecco's modified Eagle's medium (DMEM; Gibco-BRL, Gaitherburg, MD, USA) containing 10\% fetal bovine serum (FBS) and 1\% penicillin/streptomycin (both from Gibco-BRL). The cells were maintained at $37^{\circ} \mathrm{C}$ in $5 \% \mathrm{CO}_{2}$ humidified atmosphere, and were subcultured at approximately $70-80 \%$ confluence in $100 \mathrm{~mm}$ diameter culture dish, and the medium was replaced every 2 days.

Preparation of PYGP. P. yezoensis was obtained from the Republic of Korea in 2014 (Suhyup, Busan, South Korea). $P$. yezoensis powder (40 g) was diluted with 1 liter distilled water and stirred for $4 \mathrm{~h}$ at room temperature. The solution was centrifuged at $3000 \mathrm{x} \mathrm{g}, 4^{\circ} \mathrm{C}$ for $20 \mathrm{~min}$ and vacuum filtered, and triple volumes of ethanol (supernatant 1:ethanol 3) were added. After $24 \mathrm{~h}$, the solution was filtered and concentrated by rotary evaporation at $40^{\circ} \mathrm{C}$. The concentrated solution was divided into $1.5 \mathrm{ml}$ tubes, freeze-dried and stored at $-70^{\circ} \mathrm{C}$ until further use.

Cell treatment. The cells were treated with PYGP $(2.5,5,10,20$ and $40 \mu \mathrm{g} / \mathrm{ml}$ ) for $24 \mathrm{~h}$ and then stimulated with $1 \mu \mathrm{g} / \mathrm{ml} \mathrm{LPS}$ with PYGP $(2.5,5,10,20$ and $40 \mu \mathrm{g} / \mathrm{ml})$ for $24 \mathrm{~h}$.

Determination of nitrite concentration. The nitrite concentration in the cultured medium was determined using Griess reagent (Enzo Life Sciences, Farmingdale, NY, USA). Fifty microliters of supernatant from the 96-well plates were mixed with the same volume of Griess reagent. After $30 \mathrm{~min}$, the absorbance was measured at $540 \mathrm{~nm}$ using a Benchmark Plus 10730 microplate reader (Benchmark; Bio-Rad Laboratories, Inc., Hercules, CA, USA). Phe percentage of nitrite concentration was calculated using the following formula: $\mathrm{NO}(\%)=\mathrm{AT} /$ $\mathrm{AC} \times 100$, where AC is the absorbance of the control and AT is the absorbance of the test group.

Determination of prostaglandin $E_{2}\left(P G E_{2}\right)$ levels. The levels of $\mathrm{PGE}_{2}$ in the RAW 264.7 macrophages were measured using the $\mathrm{PGE}_{2}$ express EIA kit according to the manufacturer's instructions (Cayman, Ann Arbor, MI, USA). The absorbance was measured using a microplate reader (Benchmark plus 10730; Bio-Rad Laboratories, Inc.). The percentage of $\mathrm{PGE}_{2}$ was calculated using the following formula: $\mathrm{PGE}_{2}(\%)=\mathrm{AT} /$ $\mathrm{AC} \times 100$, where $\mathrm{AC}$ is the absorbance of the control and AT is the absorbance of the test group.
Determination of ROS generation. The levels of ROS were determined using 2',7'-dichlorofluorescenin diacetate (DCF-DA; Sigma-Aldrich, St. Louis, MO, USA). The RAW 264.7 cells were incubated with DCF-DA for $30 \mathrm{~min}$ in the dark. The cells were then washed twice with ice-cold phosphate-buffered saline (PBS). The level of ROS was analyzed at an excitation wavelength of $480 \mathrm{~nm}$ and an emission wavelength of $535 \mathrm{~nm}$ using a fluorescence microplate reader (FilterMAX F5; Molecular Devices, LLC, Sunnyvale, CA, USA). The percentage of ROS was calculated as follows: $\operatorname{ROS}(\%)=\mathrm{AT} / \mathrm{AC} \times 100$, where AC is the absorbance of the control and AT is the absorbance of the test group.

Determination of thiobarbituric acid reactive substances (TBARS). The levels of TBARS in the RAW 264.7 cells were measured using the TBARS assay kit according to the manufacturer's instructions (Cell Biolabs, San Diego, CA, USA). The absorbance was measured using a microplate reader (Benchmark plus 10730; Bio-Rad Laboratories, Inc.). The percentage of TBARS was calculated as follows: TBARS $(\%)=\mathrm{AT} / \mathrm{AC} \times 100$, where AC is the absorbance of the control and AT is the absorbance of the test group.

Western blot analysis. The RAW 264.7 cells were washed with ice-cold PBS ( $0.15 \mathrm{M}$ sodium phosphate, $0.15 \mathrm{M}$ sodium chloride, $\mathrm{pH}$ 7.4; Gibco-BRL), followed by lysis buffer [150 mM sodium chloride, $50 \mathrm{mM}$ Tris- $\mathrm{HCl}$ ( $\mathrm{pH} 7.5), 0.5 \%$ sodium deoxycholate, $0.1 \%$ sodium dodecyl sulfate, $1 \%$ Triton X-100, and $2 \mathrm{mM}$ ethylenediaminetetra-acetic acid; Intron Biotechnology Inc., Seongnam, Korea] with inhibitors $\left(1 \mathrm{mM} \mathrm{Na}_{3} \mathrm{VO}_{4}, 1 \mu \mathrm{g} / \mathrm{ml}\right.$ aprotinin, $1 \mu \mathrm{g} / \mathrm{ml}$ leupeptin, $1 \mu \mathrm{g} / \mathrm{ml}$ pepstatin A and $1 \mathrm{mM}$ PMSF; Sigma-Aldrich). Protein levels were determined using the bichinchominic acid assay kit (Pierce Biotechnology, Rockford, IL, USA). Equal protein amounts $(20 \mu \mathrm{g})$ of each sample were separated by $10-15 \%$ sodium dodecyl sulfate (SDS)-polyacrylamide gel and transferred onto a polyvinylidene fluoride membrane (Millipore, Billerica, MA, USA). The transferred membrane was blocked with $1 \%$ bovine serum albumin (BSA) in TBS-T [10 mM Tris- $\mathrm{HCl}$ (pH 7.5), $150 \mathrm{mM}$ $\mathrm{NaCl}$, and $0.1 \%$ Tween-20; USB Corporation, Cleveland, $\mathrm{OH}$, USA]. Subsequently, the membrane was incubated for $4 \mathrm{~h}$ at room temperature with the following primary immunoglobulin $\mathrm{G}$ antibodies, diluted to 1:1,000 in BSA/TBS-T: rabbit anti-mouse STAT3 polyclonal antibody (sc-482), goat anti-mouse p-STAT3 polyclonal antibody (sc-7993), rabbit antimouse STAT6 polyclonal antibody (sc-621), rabbit anti-mouse p-STAT6 polyclonal antibody (sc-11762), rabbit anti-mouse CD163 polyclonal antibody (sc-33560), rabbit anti-mouse CD206 polyclonal antibody (sc-48758), rabbit anti-mouse glyceraldehyde 3-phosphate dehydrogenase (GAPDH) polyclonal antibody (sc-25778) (all from Santa Cruz Biotechnology Inc., Dallas, TX, USA). The secondary antibodies were peroxidase-conjugated anti-goat (81-1620), anti-mouse (62-6520), and anti-rabbit (65-6120) antibodies (1:10,000; Bethyl Laboratories, Montgomery, TX, USA). Antibody binding was visualized using the Super Signal West Pico Stable Peroxide Solution and the Super Signal West Pico Luminol/Enhancer solution (Thermo Fisher Scientific Inc., Rockford, IL, USA). The signal was monitored using Kodak X-ray film and a developer and fixer twin pack (both from Kodak, Rochester, NY, USA). 

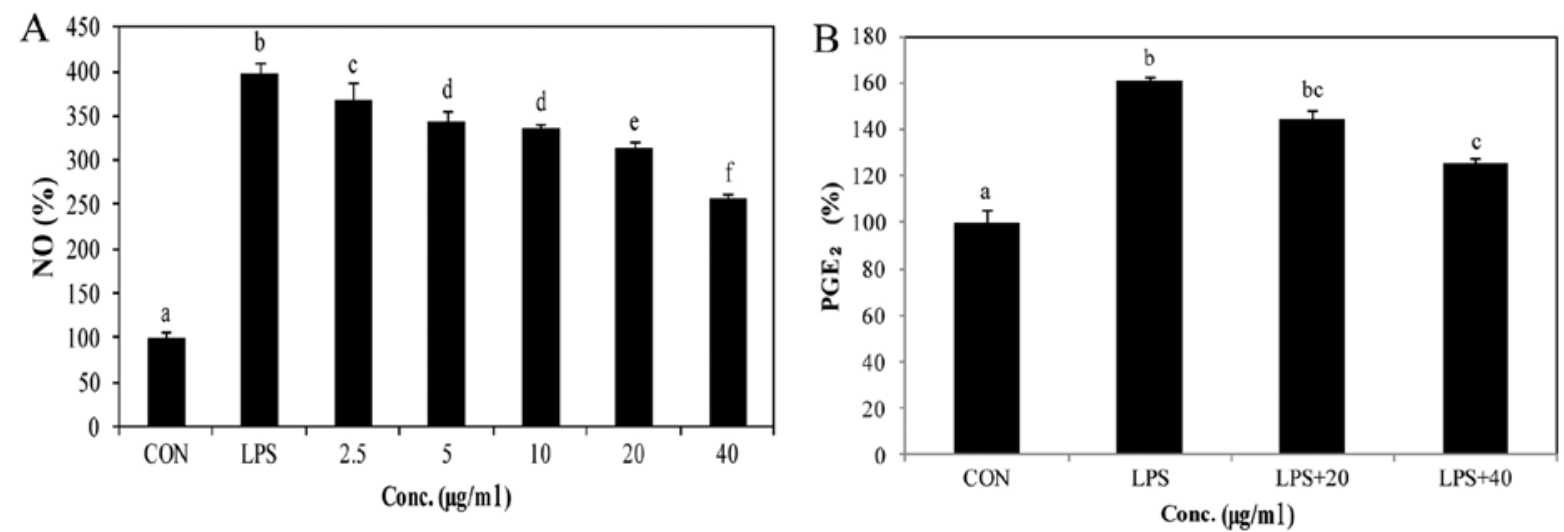

Figure 1. Effect of Pyropia yezoensis glycoprotein (PYGP) on the level of nitric oxide (NO) and prostaglandin E2 (PGE 2 ) following LPS-induced M1 activation RAW 264.7 cells were pre-treated with PYGP $(2.5,5,10,20$ and $40 \mu \mathrm{g} / \mathrm{ml})$ for $24 \mathrm{~h}$ and then administered $1 \mu \mathrm{g} / \mathrm{ml} \mathrm{LPS}$ with PYGP $(2.5,5,10,20 \mathrm{and} 40 \mu \mathrm{g} / \mathrm{ml})$ for $24 \mathrm{~h}$. (A) NO level. (B) $\mathrm{PGE}_{2}$ level. Values are presented as the means \pm standard deviation. Bars labeled with different letters indicate statistically significant differences $(\mathrm{P}<0.05)$

Reverse transcription-quantitative polymerase chain reaction $(R T-q P C R)$. Total RNA was extracted from the cells using TRIzol reagent (Invitrogen, Carlsbad, CA, USA). cDNA was sythesized using RevoScript ${ }^{\mathrm{TM}}$ RT preMix (Intron Biotechnology Inc.). The synthesized cDNA and primer were added to $2 \mathrm{X}$ TOPsimple ${ }^{\mathrm{TM}}$ DyeMIX-nTaq (Enzynomics Inc., Deajeon, Korea). Amplifications were performed using TOPreal $^{\mathrm{TM}}$ qPCR 2X PreMIX SYBR-Green (Enzynomics Inc.) in a $E_{c o}{ }^{\mathrm{TM}}$ Real-Time PCR system (Illumina Inc., San Diego, CA, USA). Gene expression levels were normalized to GAPDH and calculated using the comparative $\Delta \Delta C T$ method, as previously described (18). The oligonucleotide primers used for PCR were as follows: IL-12 forward, 5'-CGT GCT CAT GGC TGG TGC AAA-3' and reverse, 5'-CTT CAT CTG CAA GTT CTT GGG-3'; IFN- $\gamma$, forward, 5'-ACA CTC ATT GAA AGC CTA GAA AGT CTG-3' and reverse, 5'-ATT CTT CTT ATT GGC ACA CTC TCT ACC-3'; IL-6 forward, 5'-GTT CTC TGG GAA ATC GTG GA-3' and reverse, 5'-TGT ACT CCA GGT AGC TAT GG-3'; nitric oxide synthase-2 (NOS-2) forward, 5'-CTG CAT GGA ACA GTA TAA GGC AAA C-3' and reverse, 5'-CAG ACA GTT TCT GGT CGA TGT CAT GA-3'; IL-1 $\beta$ forward, 5'-GTG TGG ATC CCA AGC AAT ACC CA-3' and reverse, 5'-CCA GCC CAT ACT TTA GGA AGA CAC AGA-3'; Ym1 forward, 5'-GGA TGG CTA CAC TGG AGA AA-3' and reverse, 5'-AGA AGG GTC ACT CAG GAT AA-3'; FIZZ1 forward, 5'-CCC TCC ACT GTA ACG AAG-3' and reverse, 5'-GTG GTC CAG TCA ACG AGT AA-3'; ARG1 forward, 5'-CTC CAA GCC AAA GTC CTT AGA G-3' and reverse, 5'-AGG AGC TGT CAT TAG GGA CAT C-3'; IL-10 forward, 5'-CTG CTC CAC TGC CTT GCT CTT ATT-3' and reverse, 5'-GTG AAG ACT TTC TTTCAA ACA AAG-3'; Krüppel-like factor 4(KLF4) forward, 5'-GCA CAC CTG CGA ACT CAC AC-3' and reverse, 5'-CCG TCC CAG TCA CAG TGG TAA-3'; peroxisome proliferator-activated receptor $\gamma(\mathrm{PPAR} \gamma)$ forward, 5'-ACC ACT CGC ATT CCT TTG AC3' and reverse, 5'-AAC CAT TGG GTC AGC TCT TG-3'; GAPDH forward, 5'-ACT CCA CTC ACG GCA AAT TCA-3' and reverse, 5'-CGC TCC TGG AAG ATG GTG-3'.

Small interfering RNA (siRNA) transfection. STAT3 (5'-CCC GCC AAC AAA UUA AGA ATT-3' and 3'-UUC UUA AUU UGU UGG CGG GTT-5') and STAT6 (5'-CCA AGA CAA
CAA CGC CAA ATT-3' and 3'-UUU GGC GUU GUU GUC UUG GTT-5') and silencer negative control siRNAs were purchased from GenePharma (Shanghai, China). It should be noted that initially, we purchased 3 types of siRNAs for STAT3 and STAT6 (siRNA-1-3, respectively). From these siRNAs, STAT3 siRNA-3 and STAT6 siRNA-3 most effectively suppressed the expression of STAT3 and STAT6, respectively. Thus, these siRNAs were recorded and used in our experiments. The RAW 264.7 cells were transiently transfected with siRNA for $24 \mathrm{~h}$ using Lipofectamine 2000 reagent (Invitrogen), according to the manufacturer's instructions. Following transfection, the medium was replaced with fresh culture medium.

Statistical analysis. Values are presented as the means \pm standard deviation and data were analyzed with SPSS ver. 10.0 software (SPSS Inc., Chicago, IL, USA) using an analysis of variance followed by a Duncan's multiple range test. P-values $<0.05$ were considered to indicate statistically significant differences.

\section{Results}

Effect of PYGP on LPS-induced NO release. We used Griess reagent to determine the production of NO (Fig. 1A). In the macrophages stimulated with LPS, NO production was significantly increased, and NO was released into the extracellular matrix. However, the NO concentration was high in the culture medium only in the LPS only-treated group. In the presence of PYGP, the concentration of NO in the culture medium was inhibited in a dose-dependent manner (Fig. 1A).

Effect of PYGP on LPS-induced PGE $E_{2}$ production. The production levels of $\mathrm{PGE}_{2}$ were measured in the LPS-stimulated RAW 264.7 cells (Fig. 1B). GGE $_{2}$ secretion into the supernatant of the cell cultures was estimated by $\mathrm{PGE}_{2}$ express ELISA kit. Following stimulation with LPS $(1 \mu \mathrm{g} / \mathrm{ml}), \mathrm{PGE}_{2}$ expression in the medium was markedly increased. However, when the RAW 264.7 cells were pre-treated with PYGP, $\mathrm{PGE}_{2}$ expression was significantly decreased.

Effect of PYGP on LPS-induced TBARS and ROS generation. TBARS formation was determined in the LPS-stimulated 

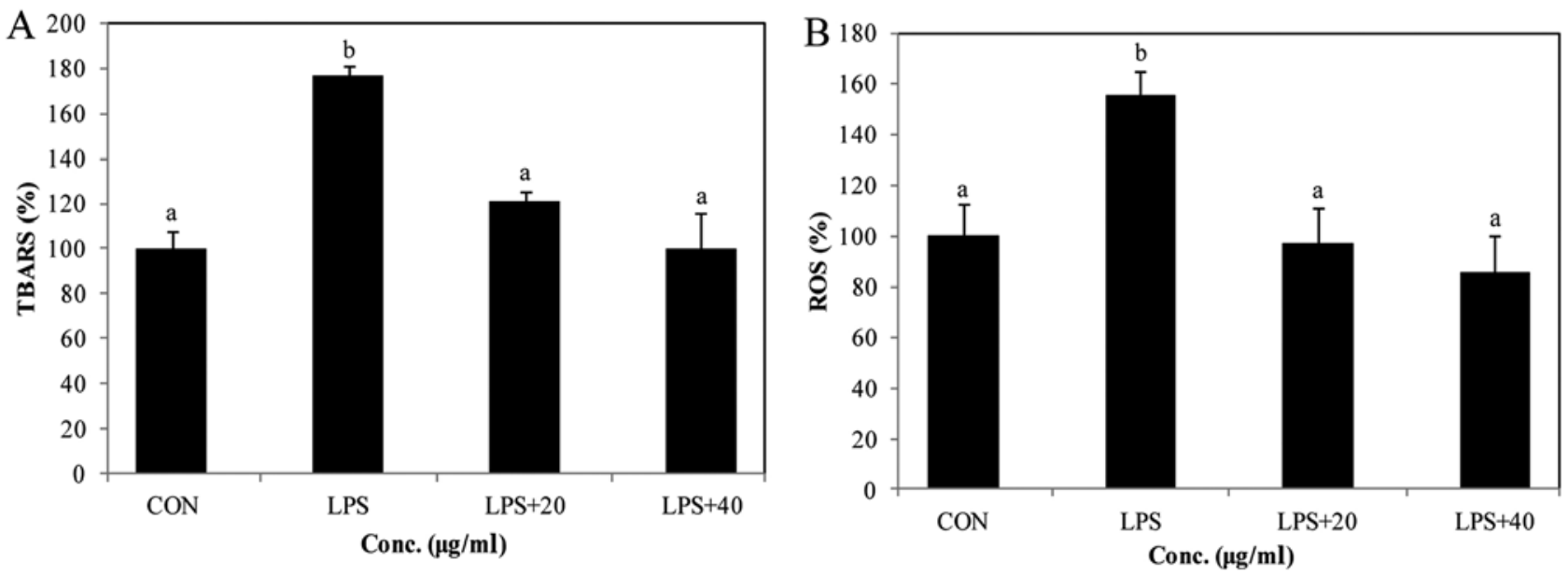

Figure 2. Effect of Pyropia yezoensis glycoprotein (PYGP) on the levels of TBARS and ROS following LPS-induced M1 activation. RAW 264.7 cells were pre-treated with PYGP (20 and $40 \mu \mathrm{g} / \mathrm{ml})$ for $24 \mathrm{~h}$ and then administered $1 \mu \mathrm{g} / \mathrm{ml} \mathrm{LPS} \mathrm{with} \mathrm{PYGP} \mathrm{(20} \mathrm{and} 40 \mu \mathrm{g} / \mathrm{ml})$ for $24 \mathrm{~h}$. (A) TBARS levels. (B) ROS levels. Values are presented as the means \pm standard deviation. Bars labeled with different letters indicate statistically significant differences $(\mathrm{P}<0.05)$.
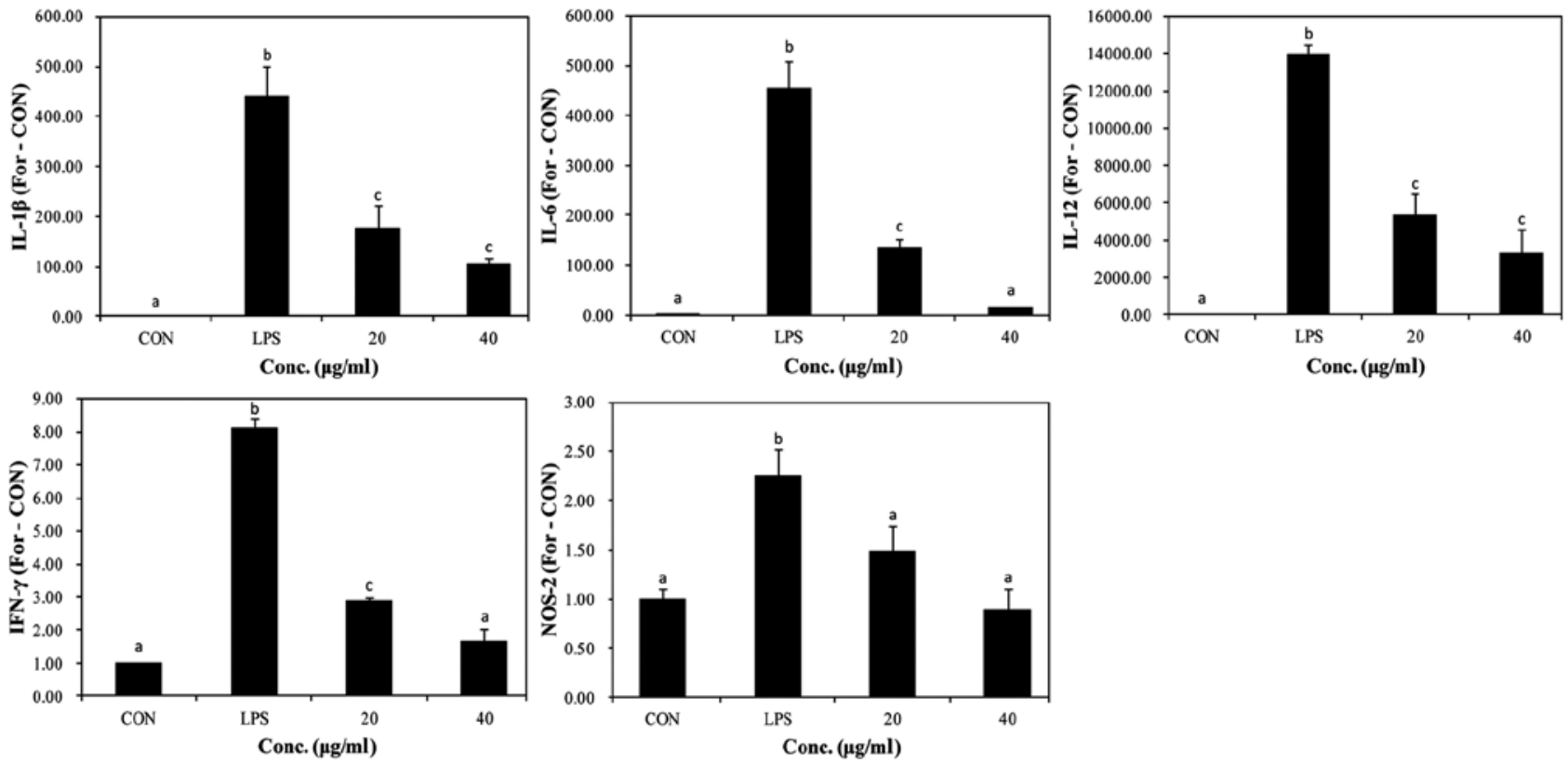

Figure 3. Effect of Pyropia yezoensis glycoprotein (PYGP) on the levels of M1 markers following LPS-induced M1 activation. RAW 264.7 cells were pre-treated with PYGP $(20$ and $40 \mu \mathrm{g} / \mathrm{ml})$ for $24 \mathrm{~h}$ and then administered $1 \mu \mathrm{g} / \mathrm{ml} \mathrm{LPS} \mathrm{with} \mathrm{PYGP}(20$ and $40 \mu \mathrm{g} / \mathrm{ml})$ for $24 \mathrm{~h}$. Values are presented as the means \pm standard deviation. Bars labeled with different letters indicate statistically significant differences $(\mathrm{P}<0.05)$.

RAW 264.7 cells through oxidative stress mechanisms (Fig. 2A). Our results revealed that the TBARS levels in the cells were significantly higher in the LPS only-treated group. In addition, pret-reatment with PYGP significantly decreased the TBARS levels in the RAW 264.7 cells in comparison with the LPS onlytreated group.

We also wished to determine whether PYGP attenuates ROS generation in LPS-stimulated RAW 264.7 cells using DCF-DA (Fig. 2B). The LPS-stimulated RAW 264.7 cells significantly generated ROS compared with the unstimulated controls. However, pre-treatment with PYGP markedly decreased the generation of ROS induced by LPS.

Effects of PYGP on M1 polarization markers. It is known that M1-activated RAW 264.7 macrophages produce pro-inflam- matory cytokines. Thus, in our study, in order to examine the effects of PYGP on the LPS-stimulated RAW 264.7 cells, we determined the mRNA expression of pro-inflammatory cytokines (Fig. 3). The results of RT-qPCR revealed that LPS upregulated the mRNA expression of the pro-inflammatory cytokines, IL-1 $\beta$, IL-6, IL-12, IFN- $\gamma$ and NOS-2. Pre-treatment with PYGP significantly suppressed the mRNA expression of these pro-inflammatory cytokines. These results suggest that PYGP suppresses pro-inflammatory cytokine expression and prevents the M1 activation of LPS-stimulated RAW 264.7 macrophages.

Effects of PYGP on M2 polarization markers. M2-activated RAW 264.7 macrophages lead to metabolic alterations. Consequently, macrophages produce Ym1, ARG1, IL-10 and 

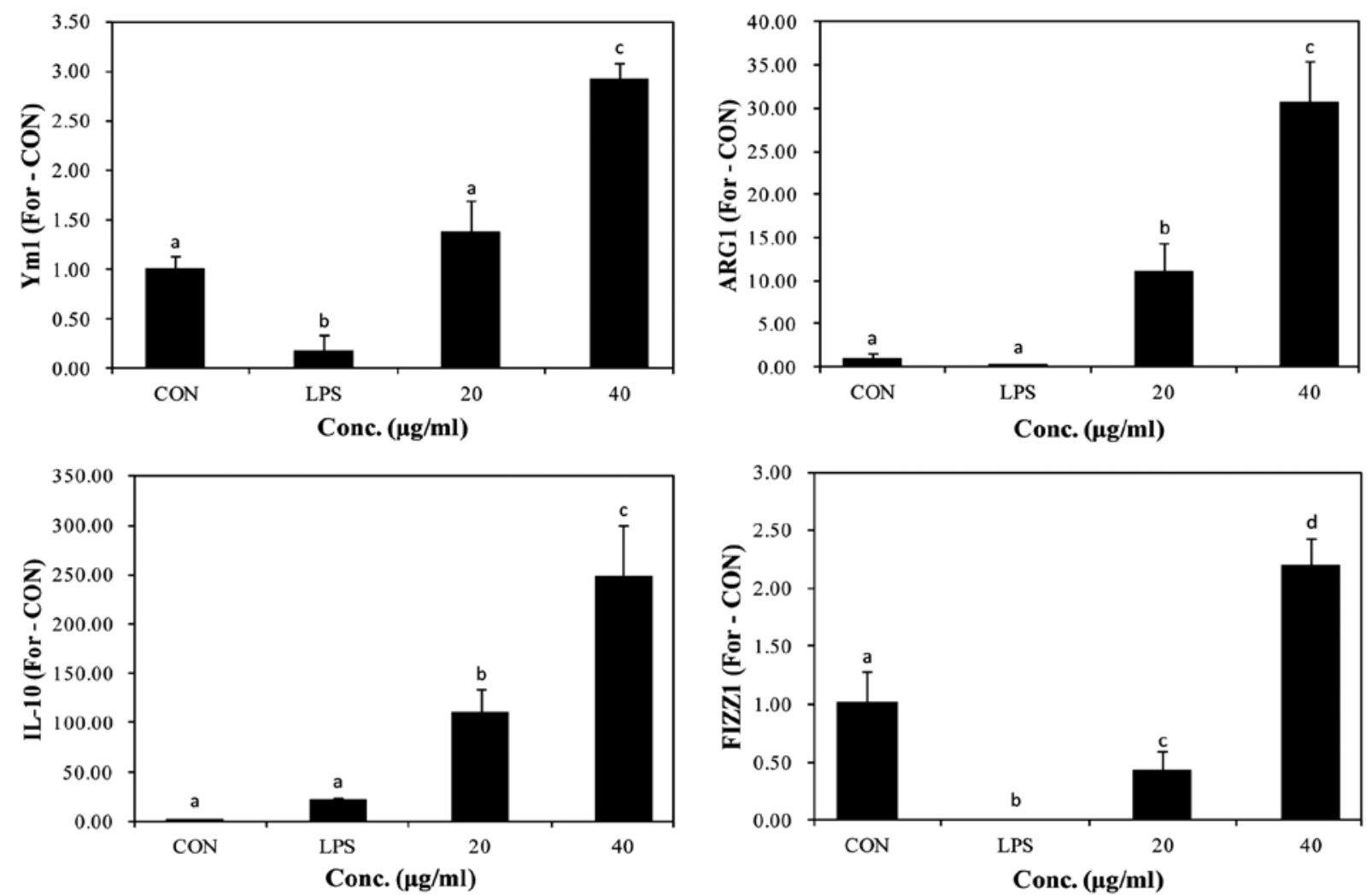

Figure 4. Effect of Pyropia yezoensis glycoprotein (PYGP) on the levels of M2 markers following LPS-induced M1 activation. RAW 264.7 cells were pre-treated with PYGP $(20$ and $40 \mu \mathrm{g} / \mathrm{ml})$ for $24 \mathrm{~h}$ and then administered $1 \mu \mathrm{g} / \mathrm{ml}$ LPS with PYGP $(20$ and $40 \mu \mathrm{g} / \mathrm{ml})$ for $24 \mathrm{~h}$. Values are presented as the means \pm standard deviation. Bars labeled with different letters indicate statistically significant differences $(\mathrm{P}<0.05)$.

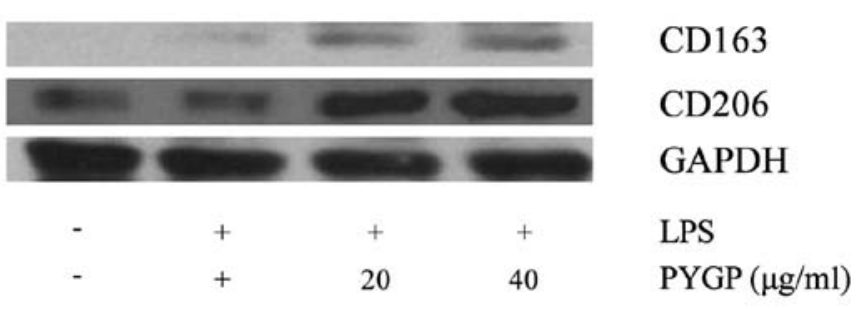

Figure 5. Effect of Pyropia yezoensis glycoprotein (PYGP) on the levels of the CD163 and CD206 following LPS-induced M1 activation. RAW 264.7 cells were pre-treated with PYGP $(20$ and $40 \mu \mathrm{g} / \mathrm{ml})$ for $24 \mathrm{~h}$ and then administered $1 \mu \mathrm{g} / \mathrm{ml}$ LPS with PYGP $(20$ and $40 \mu \mathrm{g} / \mathrm{ml})$ for $24 \mathrm{~h}$.

FIZZ1. The mRNA expression of M2 markers was not observed in the control group and LPS-stimulated. However, in the PYGP-treated cells, the mRNA expression of M2 marker genes increased (Fig. 4). These results indicate that PYGP prevents the induction of the M1 macrophage phenotype by LPS and promotes the switch to the M2 phenotype.

There is evidence to indicate that non-opsonic receptors, such as CD163 and CD206 are upregulated M2-activated macrophages $(38,39)$. In this study, we used western blot analysis to measure the protein expression levels of CD163 and CD206 (Fig. 5). Stimulation with LPS decreased CD163 and CD206 expression in the RAW 264.7 cells. In the cells pretreated with PYGP, the expression levels of CD163 and CD206 were significantly increased compared with those of the control and the LPS only-treated groups.

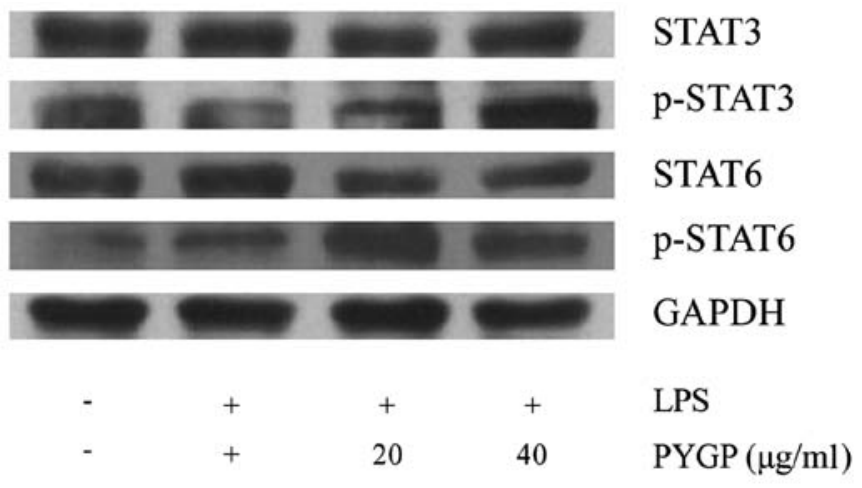

Figure 6. Effect of Pyropia yezoensis glycoprotein (PYGP) on the expression of STAT3 and STAT6 following LPS-induced M1 activation. RAW 264.7 cells were pre-treated with PYGP $(20$ and $40 \mu \mathrm{g} / \mathrm{ml})$ for $24 \mathrm{~h}$ and then administered $1 \mu \mathrm{g} / \mathrm{ml}$ LPS with PYGP (20 and $40 \mu \mathrm{g} / \mathrm{ml}$ ) for $24 \mathrm{~h}$.

Effects of PYGP on the STAT3 and STAT6 signaling pathways. STAT3 and STAT6 are well known transcription factors that induce M2 macrophage activation and inhibit inflammation. Thus, we measured the phosphorylation levels of STAT3 and STAT6 by western blot analysis (Fig. 6). Our results revealed that stimulation with LPS did not affect STAT3 and STAT6 phosphorylation. In the PYGP-pre-treated cells, the phosphorylation levels of STAT3 and STAT6 were increased in a dose-dependent manner. However, the total STAT3 and STAT6 protein expression levels were not altered following treatment with LPS or pre-treatment with PYGP. 

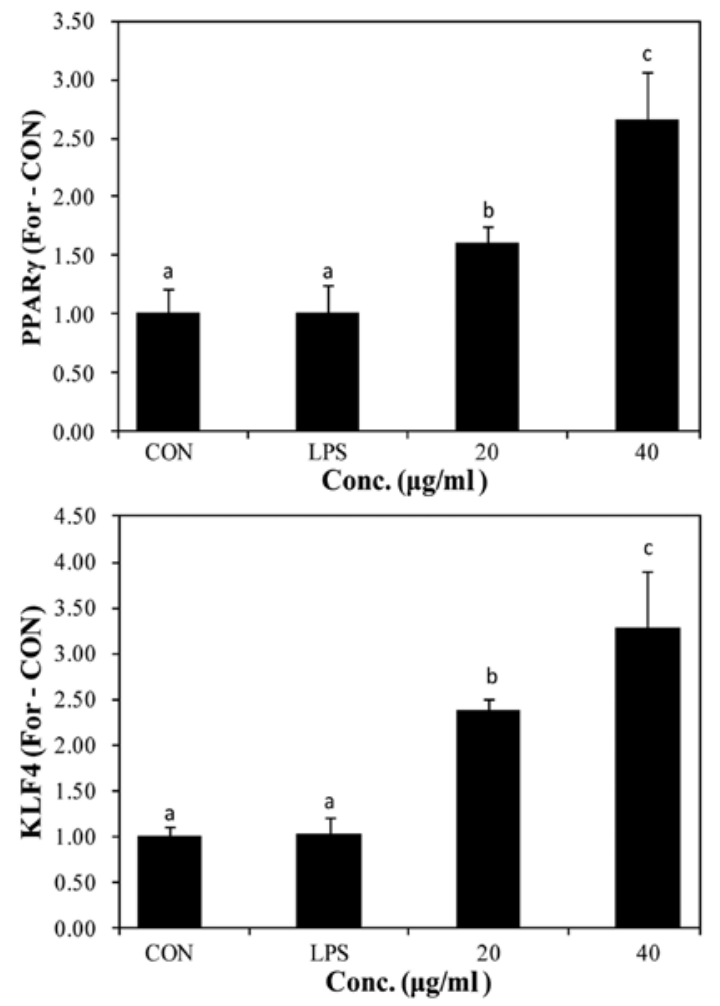

Figure 7. Effect of Pyropia yezoensis glycoprotein (PYGP) on the mRNA expression of PPAR $\gamma$ and KLF4 following LPS-induced M1 activation. RAW 264.7 cells were pre-treated with PYGP (20 and $40 \mu \mathrm{g} / \mathrm{ml})$ for $24 \mathrm{~h}$ and then administered $1 \mu \mathrm{g} / \mathrm{ml}$ LPS with PYGP $(20$ and $40 \mu \mathrm{g} / \mathrm{ml})$ for $24 \mathrm{~h}$. Values are presented as the means \pm standard deviation. Bars labeled with different letters indicate statistically significant differences $(\mathrm{P}<0.05)$.

The expression of PPAR $\gamma$ and KLF4 has been reported to increase by STAT6 phosphorylation. The increased expression of PPAR $\gamma$ and KLF4 promotes the switch to the M2 macrophage phenotype $(19,20)$. We used RT-qPCR to determine the mRNA expression of PPAR $\gamma$ and KLF4 following stimulation with LPS and pre-treatment with PYGP (Fig. 7). Stimulation with LPS did not affect PPAR $\gamma$ and KLF4 mRNA expression compared with the control group. However, pre-treatment with PYGP increased PPAR $\gamma$ and KLF4 mRNA expression in a dose-dependent manner.

Analysis of M2 polarization markers following transfection with siRNA targeting STAT3 and STAT6. STAT3 and STAT6 are major transcription factors that are involved in the regulation of the immune response by macrophages. STAT3 and STAT6 are essential for macrophage differentiation into the M2 phenotype $(23,43)$. In this study, to clarify whether STAT3 and STAT6 play a role in the PYGP-mediated M2 activation of RAW 264.7 cells, we examined the expression of M2 markers following the knockdown of STAT3 and STAT6 gene expression. We tested 3 types of STAT3 and STAT6 siRNA, and the expression of STAT3 and STAT6 was most significantly downregulated using STAT3 siRNA-3 and STAT6 siRNA-3, respectively (Fig. 8). Transfection of the RAW 264.7 cells with STAT3 siRNA and STAT6 siRNA attenuated the PYGPinduced increase in the mRNA expression of FIZZ1, Ym1 and ARG1 (Figs. 9 and 10). These results indicate that the activation of STAT3 and STAT6 plays an important role in the switch

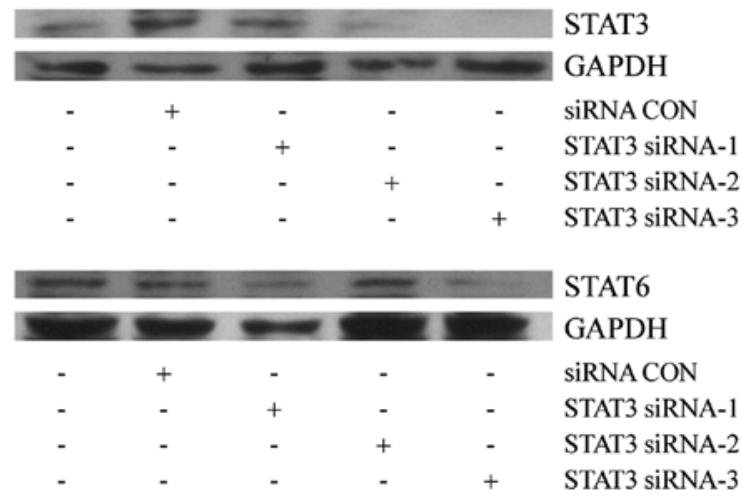

Figure 8. Effect of STAT3 siRNA and STAT6 siRNA on the expression of STAT3 and STAT6 in RAW 264.7 cells. RAW 264.7 cells were transfected with STAT3 siRNA and STAT6 usihng Lipofectamine for $12 \mathrm{~h}$.

from the M1 to the M2 RAW 264.7 macrophage phenotype induced by PYGP.

\section{Discussion}

Macrophages play a key role in the early stages of the adaptive immune reponse, the innate immune response and in the regulation of inflammation. Macrophages can differentiate into different phenotypes, namely the M0 (unstimulated state), M1 (classical activation) and the M2 (alterative activation) phenotype (21). IFN, TNF, GM-CSF and TLR ligand stimulation promotes the production of pro-inflammatory cytokines, such as IL-1 $\beta$, IL-6, TNF- $\beta$, IL-12, IFN- $\gamma$. Moreover, it promotes the switch to the M1 macrophage phenotype $(22,23)$. IL-4, IL-13, IL-10, glucocorticoids and M-CSF stimulation promotes the secretion of IL-1ra, IL-10, TGF- $\beta$ and the switch to the M2 macrophage phenotype (24).

LPS is a major stimulator of the M1 macrophage phenotype via TLR4 stimulation in (25). LPS-activated M1 macrophages produce $\mathrm{NO}$ and $\mathrm{PGE}_{2}$, and protect the host against infections. However, the abnormal and chronic production of $\mathrm{NO}$ and $\mathrm{PGE}_{2}$ leads to the development of various diseases $(26,27)$. In this study, we demonstrated that stimulation with LPS significantly increased $\mathrm{NO}$ and $\mathrm{PGE}_{2}$ production, which were inhibited by pre-treatment of the RAW 264.7 cells with PYGP.

The classical activation of M1 macrophages increases aerobic glycolysis, glucose uptake and the conversion of pyruvate to lactate $(28,29)$. Moreover, ROS production is increased from the mitochondria via NADPH oxidase activation (30). Lipid peroxidation-produced ROS cause cellular injury by the inactivation of membrane enzymes and receptors (31). In the present study, the levels of ROS and TBARS were increased in the LPS treatment group compared with the control group. However, pre-treatment with PYGP significantly decreased the LPS-induced production of ROS and TBARS.

M1 and M2 macrophages do not only differ in their biological functions, but also as regards metabolism. The main differentiation between M1 and M2 macrophages is L-arginine metabolism. L-arginine has three metabolic pathways, including NO production by NOS-2, ureum and L-ornithine by arginase and agmatine by arginine decarboxylase $(29,32,33)$. These characteristics can be utilized in macrophages in the active state. Lipid 

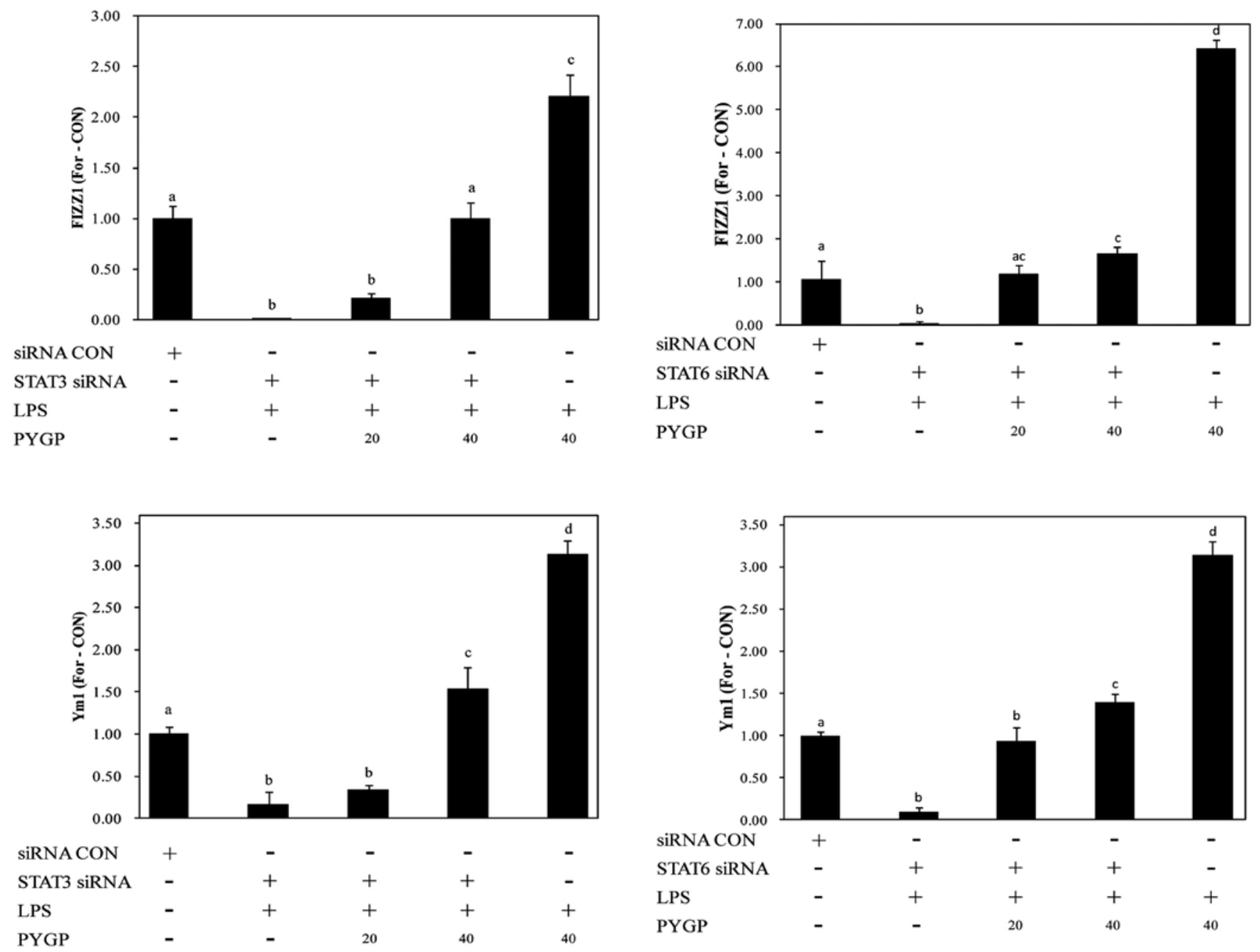

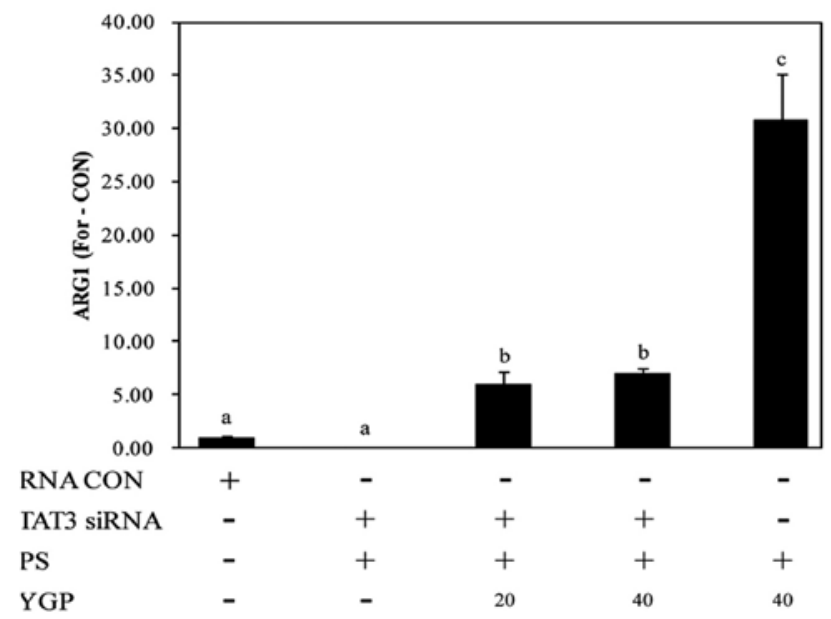

Figure 9. Effect of Pyropia yezoensis glycoprotein (PYGP) on the levels of M2 markers following LPS-induced M1 activation in transfected RAW 264.7 cells. The cells were transfected with STAT3 siRNA using Lipofectamine. After transfection, the cells were pre-treated with PYGP $(20$ and $40 \mu \mathrm{g} / \mathrm{ml})$ for $24 \mathrm{~h}$ and then administered $1 \mu \mathrm{g} / \mathrm{ml}$ LPS with PYGP $(20$ and $40 \mu \mathrm{g} / \mathrm{ml})$ for $24 \mathrm{~h}$. Values are presented as the means \pm standard deviation. Bars labeled with different letters indicate statistically significant differences $(\mathrm{P}<0.05)$.

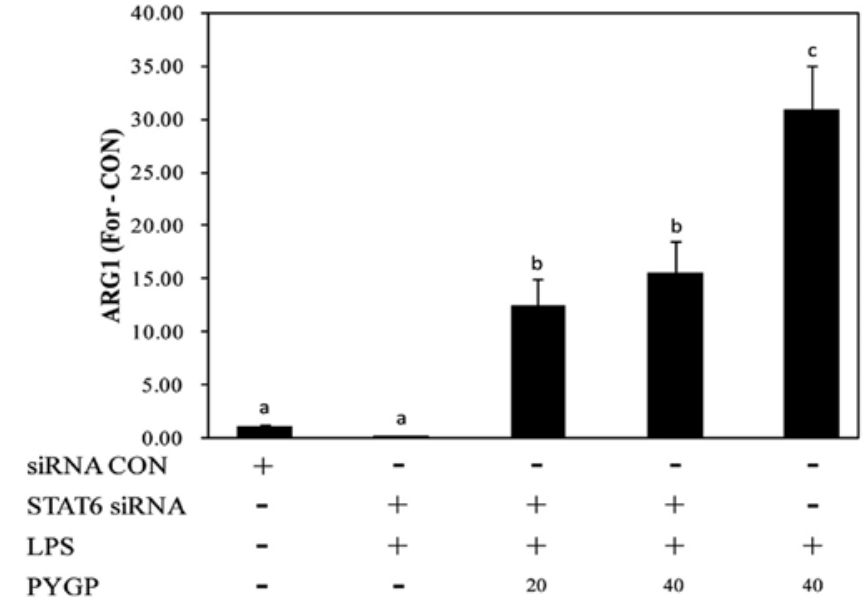

Figure 10. Effect of Pyropia yezoensis glycoprotein (PYGP) on the levels of M2 markers following LPS-induced M1 activation in transfected RAW 264.7 cells. The cells were transfected with STAT6 siRNA using Lipofectamine. After transfection, the cells were pre-treated with PYGP $(20$ and $40 \mu \mathrm{g} / \mathrm{ml})$ for $24 \mathrm{~h}$ and then administered $1 \mu \mathrm{g} / \mathrm{ml}$ LPS with PYGP $(20$ and $40 \mu \mathrm{g} / \mathrm{ml})$ for $24 \mathrm{~h}$. Values are presented as the means \pm standard deviation. Bars labeled with different letters indicate statistically significant differences $(\mathrm{P}<0.05)$. metabolism also differs between M1 and M2 macrophages. This differentiation is revealed by the transcriptional profiling of the
IL-13-steered human monocyte (34). The function of these genes is not yet fully understood, such as that of FIZZ. PPAR ligation 
has been reported to inhibit the expression of pro-inflammatory cytokines and NOS-2 (35,36). Futhermore, differences in cytokine secretion have been observed between M1 and M2 macrophages. M1 secrete pro-inflammatory cytokines, such as IL-1 $\beta$, IL-6, IL-12 and type 1 IFN, whereas M2 secrete anti-inflammatory cytokines, such as IL-10 and TGF- $\beta$ (37). In addition, IL-4, IL-13 and IL-10 upregulate several non-opsonic receptors, such as mannose receptor (CD206) and CD163 $(38,39)$. These features have been used in many studies as markers to distinguish between the activity of macrophages (40-42). In the present study, LPS increased the production of pro-inflammatory cytokines, including IL-1 $\beta$, IL-6, IL-12, IFN- $\gamma$ and NOS-2. However, pre-treatment with PYGP inhibited these pro-inflammatory cytokines and increased the expression of M2-associated markers, such as CD163, CD206, Ym1, FIZZ1 and ARG1. These results suggest that PYGP promotes the switch from the M1 to the M2 phenotype following stimulation with LPS.

M2 activation has been shown to involve various transcription factors. STAT3 and STAT6 play a key role in M2 activation $(23,43)$. STAT3 is the major anti-inflammatory mediator, mediate IL-10 transcription (44). The knockdown of STAT3 and STAT6 in mouse and human macrophages has been reported be prevent the switch to the M2 phenotype $(7,45,46)$. In the present study, the silencing of STAT3 and STAT6 inhibited the promoting effects of PYGP on the mRNA expression of M2 activation markers, including FIZZ1, Ym1 and ARG1. According to our observation, STAT3 siRNA and STAT6 siRNA decreased STAT3, STAT6, FIZZ1, Ym1 and ARG1 mRNA expression in the PYGP-treated M1 macrophages, indicating that the pre-treatment of M1 activated macrophages with PYGP promotes the switch to the M2 macrophage phenotype via STAT3 and STAT6 signaling.

In conclusion, our results demonstrate that stimulation with LPS activates M1 macrophages. PYGP inhibits the production of pro-inflammatory cytokines and promotes the switch to the M2 phenotype via STAT3 and STAT6 activation. These findings may provide a molecular basis for the use of PYGP as a treatment agent for LPS-induced inflammatory diseases.

\section{Acknowledgements}

This study was supported by the Research Program through the National Research Foundation of Korea (NRF) funded by the Ministry of Education (grant no. 2012R1A6A1028677).

\section{References}

1. Tugal D, Liao X and Jain MK: Transcriptional control of macrophage polarization. Arterioscler Thromb Vasc Biol 33: 1135-1144, 2013.

2. Lin SL, Li B, Rao S, Yeo EJ, Hudson TE, Nowlin BT, Pei H, Chen L, Zheng JJ, Carroll TJ, et al: Macrophage Wnt7b is critical for kidney repair and regeneration. Proc Natl Acad Sci USA 107: 4194-4199, 2010.

3. Nikolic-Paterson DJ and Atkins RC: The role of macrophages in glomerulonephritis. Nephrol Dial Transplant 16 (Suppl 5): 3-7, 2001.

4. Aliprantis AO, Diez-Roux G, Mulder LC, Zychlinsky A and Lang RA: Do macrophages kill through apoptosis? Immunol Today 17: 573-576, 1996.

5. Murray PJ and Wynn TA: Protective and pathogenic functions of macrophage subsets. Nat Rev Immunol 11: 723-737, 2011.

6. Barros MHM, Hauck F, Dreyer JH, Kempkes B and Niedobitek G: Macrophage polarisation: An immunohistochemical approach for identifying M1 and M2 macrophages. PLoS One 8: e80908, 2013.
7. Gordon S and Martinez FO: Alternative activation of macrophages: Mechanism and functions. Immunity 32: 593-604, 2010.

8. Kohchi C, Inagawa H, Nishizawa T and Soma G: ROS and innate immunity. Anticancer Res 29: 817-821, 2009.

9. Schroder K, Sweet MJ and Hume DA: Signal integration between IFNgamma and TLR signalling pathways in macrophages. Immunobiology 211: 511-524, 2006.

10. Martinez FO and Gordon S: The M1 and M2 paradigm of macrophage activation: Time for reassessment. F1000Prime Rep 6: 13, 2014.

11. Hu X and Ivashkiv LB: Cross-regulation of signaling pathways by interferon- $\gamma$ : Implications for immune responses and autoimmune diseases. Immunity 31: 539-550, 2009.

12. Mantovani A, Biswas SK, Galdiero MR, Sica A and Locati M: Macrophage plasticity and polarization in tissue repair and remodelling. J Pathol 229: 176-185, 2013.

13. Mantovani A, Garlanda C and Locati M: Macrophage diversity and polarization in atherosclerosis: A question of balance. Arterioscler Thromb Vasc Biol 29: 1419-1423, 2009.

14. Kim SC, Lee JR and Park SJ: Porphyra tenera induces apoptosis of oral cancer cells. Kor J Herbology 30: 25-30, 2015.

15. Kim YM: In JP, and Park JH: Angiotensin I converting enzyme (ACE) inhibitory activities of laver (Porphyra tenera) protein hydrolysates. Prev Nutr Food Sci 18: 11-18, 2005.

16. Shin ES, Hwang HJ, Kim IH and Nam TJ: A glycoprotein from Porphyra yezoensis produces anti-inflammatory effects in liposaccharide-stimulated macrophages via the TLR4 signaling pathway. Int J Mol Med 28: 809-815, 2011.

17. Hwang HJ, Kwon MJ, Kim IH and Nam TJ: Chemoprotective effects of a protein from the red algae Porphyra yezoensis on acetaminophen-induced liver injury in rats. Phytother Res 22: 1149-1153, 2008.

18. Livak KJ and Schmittgen TD: Analysis of relative gene expression data using real-time quantitative PCR and the $2^{-\Delta \Delta C T}$ method. Methods 25: 402-408, 2001.

19. Liao X, Sharma N, Kapadia F, Zhou G, Lu Y, Hong H, Paruchuri K, Mahabeleshwar GH, Dalmas E, Venteclef N, et al: Krüppel-like factor 4 regulates macrophage polarization. J Clin Invest 121: 2736-2749, 2011.

20. Szanto A, Balint BL, Nagy ZS, Barta E, Dezso B, Pap A, Szeles L, Poliska S, Oros M, Evans RM, et al: STAT6 transcription factor is a facilitator of the nuclear receptor PPAR $\gamma$-regulated gene expression in macrophages and dendritic cells. Immunity 33 : 699-712, 2010.

21. Labonte AC, Tosello-Trampont AC and Hahn YS: The role of macrophage polarization in infectious and inflammatory diseases. Mol Cells 37: 275-285, 2014.

22. Krausgruber T, Blazek K, Smallie T, Alzabin S, Lockstone H, Sahgal N, Hussell T, Feldmann M and Udalova IA: IRF5 promotes inflammatory macrophage polarization and TH1-TH17 responses. Nat Immunol 12: 231-238, 2011.

23. Lawrence $\mathrm{T}$ and Natoli G: Transcriptional regulation of macrophage polarization: Enabling diversity with identity. Nat Rev Immunol 11: 750-761, 2011.

24. Biswas SK and Mantovani A: Macrophage plasticity and interaction with lymphocyte subsets: Cancer as a paradigm. Nat Immunol 11: 889-896, 2010.

25. Verreck FA, de Boer T, Langenberg DM, Hoeve MA, Kramer M, Vaisberg E, Kastelein R, Kolk A, de Waal-Malefyt R and Ottenhoff TH: Human IL-23-producing type 1 macrophages promote but IL-10-producing type 2 macrophages subvert immunity to (myco)bacteria. Proc Natl Acad Sci USA 101: 4560-4565, 2004.

26. Agard M, Asakrah S and Morici LA: PGE(2) suppression of innate immunity during mucosal bacterial infection. Front Cell Infect Microbiol 3: 45, 2013.

27. Kilbourn RG and Griffith OW: Overproduction of nitric oxide in cytokine-mediated and septic shock. J Natl Cancer Inst 84: 827-831, 1992.

28. Hard GC: Some biochemical aspects of the immune macrophage. Br J Exp Pathol 51: 97-105, 1970.

29. Galván-Peña S and O'Neill LA: Metabolic reprograming in macrophage polarization. Front Immunol 5: 420, 2014.

30. West AP, Brodsky IE, Rahner C, Woo DK, Erdjument-Bromage H, Tempst P, Walsh MC, Choi Y, Shadel GS and Ghosh S: TLR signalling augments macrophage bactericidal activity through mitochondrial ROS. Nature 472: 476-480, 2011.

31. Jacobson MD: Reactive oxygen species and programmed cell death. Trends Biochem Sci 21: 83-86, 1996. 
32. Corraliza IM, Soler G, Eichmann K and Modolell M: Arginase induction by suppressors of nitric oxide synthesis (IL-4, IL-10 and PGE2) in murine bone-marrow-derived macrophages. Biochem Biophys Res Commun 206: 667-673, 1995.

33. Munder M, Eichmann K and Modolell M: Alternative metabolic states in murine macrophages reflected by the nitric oxide synthase/arginase balance: Competitive regulation by $\mathrm{CD} 4^{+}$ $\mathrm{T}$ cells correlates with Th1/Th2 phenotype. J Immunol 160: $5347-5354,1998$

34. Scotton CJ, Martinez FO, Smelt MJ, Sironi M, Locati M, Mantovani A and Sozzani S: Transcriptional profiling reveals complex regulation of the monocyte IL-1 $\beta$ system by IL-13. J Immunol 174: 834-845, 2005.

35. Raes G, De Baetselier P, Noël W, Beschin A, Brombacher F and Hassanzadeh Gh G: Differential expression of FIZZ1 and Ym1 in alternatively versus classically activated macrophages. J Leukoc Biol 71: 597-602, 2002.

36. Ricote M, Welch JS and Glass CK: Regulation of macrophage gene expression by the peroxisome proliferator-activated receptor- $\gamma$. Horm Res 54: 275-280, 2000.

37. Mantovani A, Sica A, Sozzani S, Allavena P, Vecchi A and Locati M: The chemokine system in diverse forms of macrophage activation and polarization. Trends Immunol 25: 677-686, 2004.

38. Brown BN, Londono R, Tottey S, Zhang L, Kukla KA, Wolf MT, Daly KA, Reing JE and Badylak SF: Macrophage phenotype as a predictor of constructive remodeling following the implantation of biologically derived surgical mesh materials. Acta Biomater 8: 978-987, 2012

39. Lau SK, Chu PG and Weiss LM: CD163: A specific marker of macrophages in paraffin-embedded tissue samples. Am J Clin Pathol 122: 794-801, 2004
40. Chen Z, Wu C, Gu W, Klein T, Crawford R and Xiao Y: Osteogenic differentiation of bone marrow MSCs by $\beta$-tricalcium phosphate stimulating macrophages via BMP2 signalling pathway. Biomaterials 35: 1507-1518, 2014.

41. Lee AS, Jung YJ, Kim D, Nguyen-Thanh T, Kang KP, Lee S, Park SK and Kim W: SIRT2 ameliorates lipopolysaccharideinduced inflammation in macrophages. Biochem Biophys Res Commun 450: 1363-1369, 2014.

42. Lopez-Castejón G, Baroja-Mazo A and Pelegrín P: Novel macrophage polarization model: From gene expression to identification of new anti-inflammatory molecules. Cell Mol Life Sci 68: 3095-3107, 2011.

43. Wang N, Liang $\mathrm{H}$ and Zen $\mathrm{K}$ : Molecular mechanisms that influence the macrophage m1-m2 polarization balance. Front Immunol 5: 614, 2014.

44. Murray PJ: Understanding and exploiting the endogenous interleukin-10/STAT3-mediated anti-inflammatory response. Curr Opin Pharmacol 6: 379-386, 2006.

45. Mandal P, Pratt BT, Barnes M, McMullen MR and Nagy LE: Molecular mechanism for adiponectin-dependent M2 macrophage polarization: Link between the metabolic and innate immune activity of full-length adiponectin. J Biol Chem 286: 13460-13469, 2011.

46. MacKinnon AC, Farnworth SL, Hodkinson PS, Henderson NC, Atkinson KM, Leffler H, Nilsson UJ, Haslett C, Forbes SJ and Sethi T: Regulation of alternative macrophage activation by galectin-3. J Immunol 180: 2650-2658, 2008. 